\title{
Transport Mechanism of SiGe Dot MOS Tunneling Diodes
}

\author{
P.-S. Kuo, C.-H. Lin, C.-Y. Peng, Y.-C. Fu, and C. W. Liu, Senior Member, IEEE
}

\begin{abstract}
The blockage of hole transport due to excess holes in SiGe dots was observed in the MOS tunneling diodes for the first time. The five layers of self-assembled SiGe dots are separated by 74-nm Si spacers and capped with a $130-\mathrm{nm} \mathrm{Si}$. The hole tunneling current from Pt gate electrode to p-type Si dominates the inversion current at positive gate bias and is seven orders of magnitude higher than the Al gate/oxide/p-Si device. The large work function of $\mathrm{Pt}$ is responsible for the hole transport current from $\mathrm{Pt}$ to $\mathrm{p}-\mathrm{Si}$. The incorporation of SiGe dots confines the excess holes in the valence band and forms a repulsive barrier to reduce the hole transport current from $\mathrm{Pt}$ to $\mathrm{SiGe}$ dots by 2-3 orders of magnitude in comparison with the Pt/oxide/p-Si device. This repulsive barrier also reduces the hole tunneling current from $\mathrm{SiGe}$ dots to $\mathrm{Pt}$ at negative gate bias.
\end{abstract}

Index Terms-MOS tunneling diode, repulsive barrier, SiGe dots, transport mechanism.

\section{INTRODUCTION}

$\mathbf{T}$ HE METAL OXIDE Si tunneling diodes have been used as light emitters [1] and photodetectors [2]. To tune the emission wavelength of LEDs [3] and to extend the cutoff wavelength of photodetectors [4], the SiGe layer is inserted in the Si. Optical, physical, and material properties of $\mathrm{Si} / \mathrm{Ge}$ dots were reported [5] [6]. Moreover, the current transport of a MOS diode is significantly influenced by these SiGe layers. Therefore, MOS diodes with the Pt (5.65 eV) and Al (4.1 eV) gates with SiGe dots are studied, and a new transport mechanism is proposed in this letter.

\section{DEVICE FABRICATION}

The low-temperature liquid-phase-deposited (LPD) $\mathrm{SiO}_{2}$ of $2.5 \mathrm{~nm}$ (ellipsometry) was deposited on both the p-type $\mathrm{SiGe}$ dot structure and the control p-type Si. The desired Ge dots were grown by ultrahigh vacuum chemical vapor deposition (UHVCVD) on p-type (001) substrates with the resistivity of $15-25 \Omega \cdot \mathrm{cm}$. Due to $\mathrm{Si} / \mathrm{Ge}$ interdiffusion at $600{ }^{\circ} \mathrm{C}$, the $\mathrm{Ge}$ dots transformed into $\mathrm{SiGe}$ dots. The top and bottom $\mathrm{SiGe}$ dots, including the wetting layers, have about $2 \times 10^{16}$ and $1.5 \times 10^{16} \mathrm{Ge}$ atoms $/ \mathrm{cm}^{2}$, respectively. The lower density of the bottom layer is due to the extra thermal budget after the bottom dots are grown. The SiGe layers are separated by

Manuscript received March 19, 2007; revised April 12, 2007. This work was supported by the National Science Council, Taiwan, R.O.C., under Contracts 95-2221-E-002-357 and 95-2221-E-002-370. The review of this letter was arranged by Editor E. Sangiorgi.

The authors are with the Department of Electrical Engineering and Graduate Institute of Electronics Engineering, National Taiwan University, Taipei 10617, Taiwan, R.O.C. (e-mail: chee@cc.ee.ntu.tw).

Digital Object Identifier 10.1109/LED.2007.898288

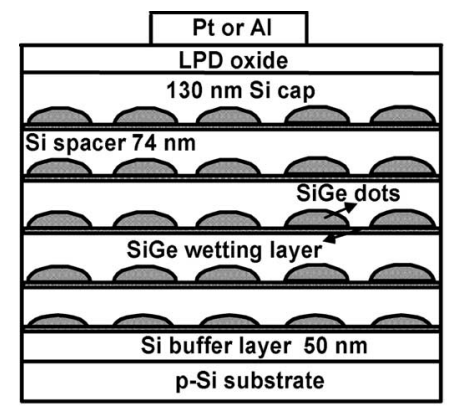

(a)

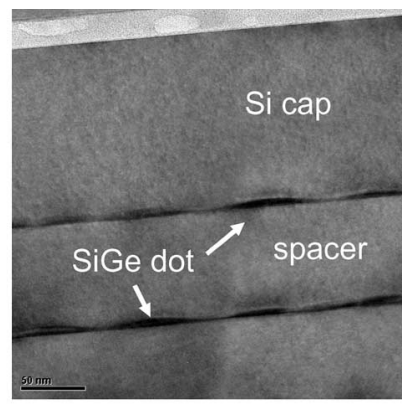

(b)
Fig. 1. (a) Structure of five-layer SiGe dot MOS diodes. (b) TEM micrograph of SiGe dot samples grown by UHVCVD.

74-nm Si spacer layers. A 130-nm Si cap was deposited on the top layer of the self-assembled SiGe dots. The SiGe dots in the top layer consist of a structure with a $\sim 100-\mathrm{nm}$ base and a $\sim 7-\mathrm{nm}$ height, with standard deviations of 13 and $3 \mathrm{~nm}$, respectively. Fig. 1 shows the schematic structure and the TEM micrograph of the five-layer SiGe dots in this letter.

\section{RESUlts And Discussion}

Due to the high density of traps in LPD oxide, the tunneling current is dominated by trap-assistant tunneling [7]. The different $\mathrm{SiGe/Si}$ structures have different magnitudes of current, indicating that $\mathrm{SiGe}$ dots play the key role in the transport mechanism and LPD oxide is not crucial. For the Pt electrode at positive gate bias, the inversion currents of single-layer and five-layer $\mathrm{SiGe}$ dot devices are two and three orders of magnitude smaller than that of control Si devices, respectively. However, for the $\mathrm{Al}$ gate electrode, five-layer $\mathrm{SiGe}$ dot device has an inversion current higher than the control Si device (Fig. 2).

Fig. 3(a) shows the band diagram of the SiGe dot devices at positive gate bias (inversion). At positive gate bias, the semiconductor is deeply depleted, and the oxide voltage is small. The large hole barrier height $(5.85 \mathrm{eV})$ of the $\mathrm{Al}$ gate prevents the hole tunneling from Al to the semiconductor. Therefore, the thermally generated electrons via the oxide/Si interface traps and the bulk traps in the depletion region dominate the gate current [8]. Due to the smaller bandgap of SiGe dots compared to $\mathrm{Si}$, the $\mathrm{SiGe}$ dot device yields a larger current. Note that due to the thin LPD oxide $(2.5 \mathrm{~nm})$ and traps in the LPD oxide, the electron tunneling (voltage dependent) through the oxide is the faster process as compared to the thermal generation process (weak voltage dependence). Therefore, the current of 


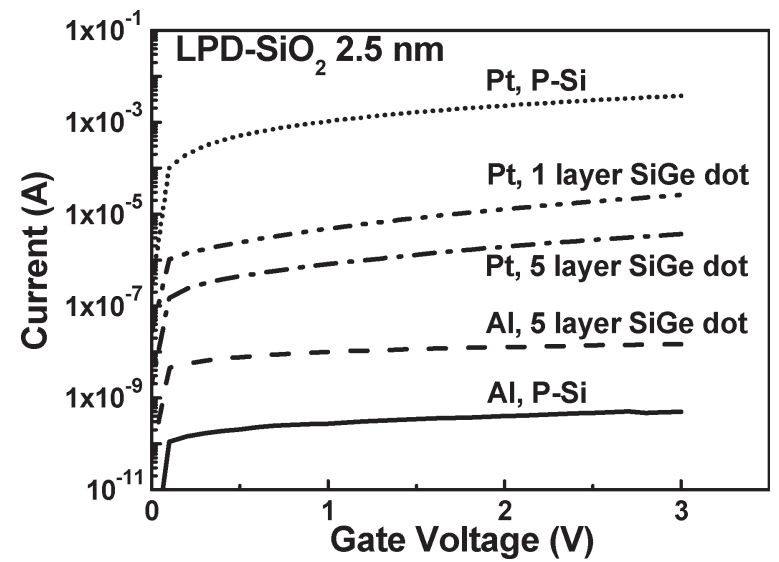

Fig. 2. $\quad I-V$ characteristics of $\mathrm{Pt}$ gate and $\mathrm{Al}$ gate devices at positive gate bias ( $\sim$ inversion). The die area is $5 \mathrm{E}-3 \mathrm{~cm}^{2}$. (a)

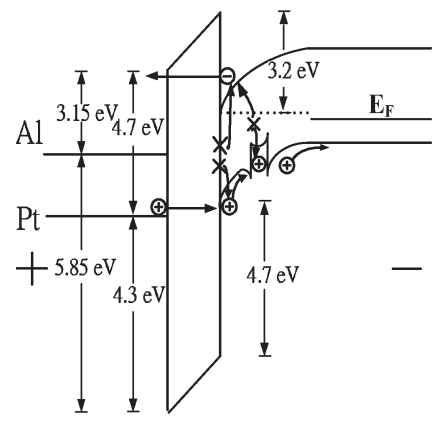

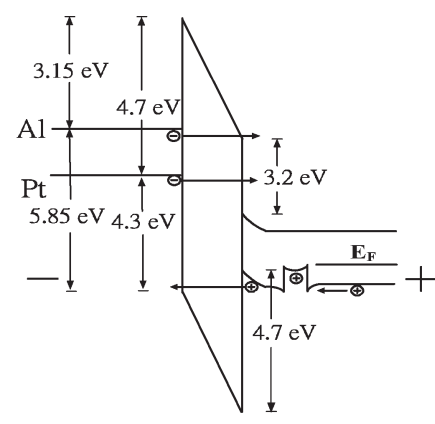

(b)
Fig. 3. Band diagrams of the NMOS SiGe dot device with $\mathrm{Pt}$ or $\mathrm{Al}$ gate operated at (a) positive gate bias ( inversion) and (b) negative gate bias ( accumulation).

Al gate devices at positive gate bias is dominated by thermally generated electrons. However, for Pt devices, the hole barrier is $4.3 \mathrm{eV}$, and the hole tunneling current from Pt to semiconductor is significantly larger than the electron generation current. As a result, the inversion current of the Pt-gate Si device is seven orders larger than the Al-gate Si device (Fig. 2) due to the additional hole tunneling current from the $\mathrm{Pt}$ gate electrode to the semiconductor. The repulsive barrier at the valence band due to the trapped holes in the SiGe dots blocks the hole transport and reduces the inversion current for the $\mathrm{Pt}$ device with $\mathrm{SiGe}$ dots. To confirm the hole blocking effect, the current at negative gate bias is also studied. For the $\mathrm{Pt}$ gate device at negative gate bias, the electron barrier from $\mathrm{Pt}$ to semiconductor is similar to the hole barrier from semiconductor to Pt (4.7 eV) [Fig. 3(b)] for both control $\mathrm{Si}$ and $\mathrm{SiGe}$ devices. The electron current tunneling from Pt to semiconductor is limited by a diffusion in p-type semiconductor, and the hole tunneling current from semiconductor to $\mathrm{Pt}$ can reach the contact rapidly by relaxation process in metal. As a result, the hole current is dominant at negative gate bias for the $\mathrm{Pt}$ device. The repulsive barrier due to the confined holes in SiGe dots blocks the hole tunneling current. Therefore, for the Pt gate, the five-layer SiGe dot device has a lower current than the Si device at the negative gate bias (Fig. 4). Note that the SiGe dots referred to in this letter include a wetting layer. The Pt gate device with a 3-nm Si cap and

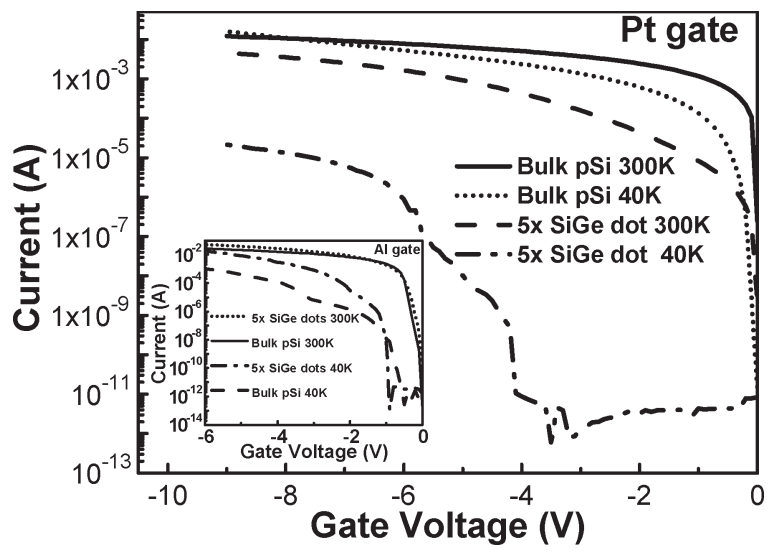

Fig. 4. Low-temperature and room-temperature $I-V$ of the $\mathrm{Si}$ and five-layer $\mathrm{SiGe}$ dot devices with $\mathrm{Pt}$ gate electrode at the negative gate bias. The die area is $5 \mathrm{E}-3 \mathrm{~cm}^{2}$. The results of $\mathrm{Al}$ gate devices are shown in the inset.

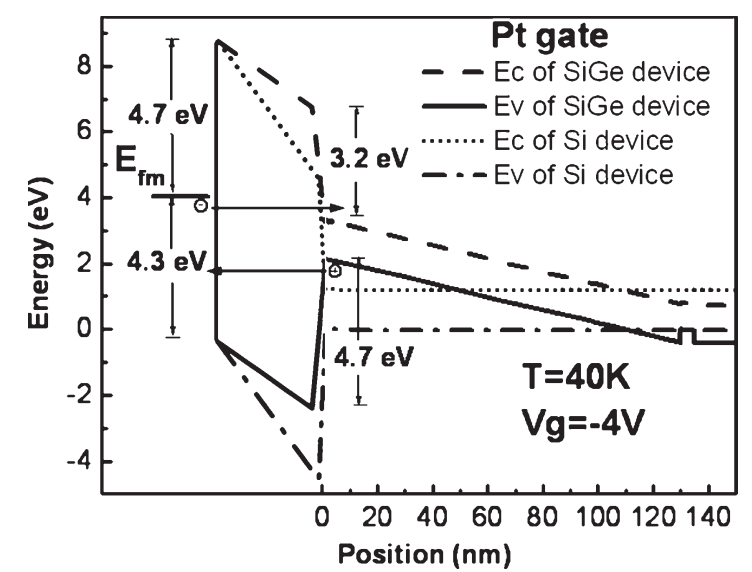

Fig. 5. Simulated band diagrams of the five-layer SiGe well device and the control Si device with $\mathrm{Pt}$ gate operated at $-4 \mathrm{~V}$ at $40 \mathrm{~K}$. The SiGe quantum wells have the thickness of $5 \mathrm{~nm}$ and are separated by 74-nm Si spacers. The Si cap is $130 \mathrm{~nm}$. The oxide thickness is $2.5 \mathrm{~nm}$ and is not scaled in the figure.

single SiGe well also has a lower current than the control Si device at both the positive and negative gate biases. The low work function $\mathrm{Al}(4.1 \mathrm{eV})$ and high work function $\mathrm{Pt}(5.65 \mathrm{eV})$ are investigated as gate electrodes to evaluate the transport mechanism of MOS tunneling diodes. Other high work function metals such as $\mathrm{Au}(5.15 \mathrm{eV})$ and ALD-TiN (5.3 eV) and low work function metals such as $\mathrm{Ti}(4.3 \mathrm{eV})$ and $\mathrm{Ta}(4.3 \mathrm{eV})$ should have similar results if Fermi level pinning does not exist. Actually, the interface states at the metal/oxide interface may cause the Fermi level pinning to some extent, which reduces the work function difference between $\mathrm{Pt}$ and Al. Therefore, the difference in characteristics between $\mathrm{Al}$ and $\mathrm{Pt}$ gate devices should be less significant if Fermi level pinning occurs. In addition, thermal treatment and process conditions can also affect the work function and should be taken into account for the device design using the hole blocking effect.

The low-temperature $(40 \mathrm{~K}) I-V$ measurement at negative gate bias is also shown in Fig. 4. Fig. 5 shows the energy band diagrams simulated by the commercial tool [9] for the $\mathrm{Pt}$ devices with five layers of 5-nm $\mathrm{Si}_{0.46} \mathrm{Ge}_{0.54}$ quantum wells as well as for the control $\mathrm{Si}$ device at $-4 \mathrm{~V}$ at $40 \mathrm{~K}$. At low temperature, more holes are confined in the SiGe quantum 
wells, and there is an additional voltage across the 130-nm $\mathrm{Si}$ cap, while for the Si MOS device, most voltage drop is across the oxide. For the five-layer $\mathrm{SiGe}$ dot device at the small negative gate bias $(|\mathrm{Vg}|<4 \mathrm{~V})$, the Fermi level of $\mathrm{Pt}$ does not exceed the conduction band edge of semiconductor at the $\mathrm{SiO}_{2} /$ semiconductor interface due to a large voltage drop across the Si cap. Therefore, the electrons in Pt are not allowed to tunnel to the substrate, and hole tunneling current from the substrate to $\mathrm{Pt}$ is dominant. When the negative gate bias increases, the Fermi level of Pt is above the conduction band edge of the semiconductor at the interface, and the electrons in Pt can tunnel to the substrate (Fig. 5). Therefore, the kink at $\sim-4 \mathrm{~V}$ in the $I-V$ curve of the Pt-gate SiGe dot device at $40 \mathrm{~K}$ is due to the onset of the electron current tunneling from Pt to semiconductor. At low temperature, due to the more abrupt transition of the Fermi-Dirac distribution, there are more holes trapped in SiGe dots. Therefore, the hole blocking effect is more significant, and the current of the Pt-SiGe dot device is smaller at $40 \mathrm{~K}$ than at room temperature. The quantum mechanism simulation shows that the hole concentration $(9.3 \times$ $\left.10^{17} \mathrm{~cm}^{-3}\right)$ at $40 \mathrm{~K}$ is 1.6 times of that $\left(6 \times 10^{17} \mathrm{~cm}^{-3}\right)$ at room temperature at $-1 \mathrm{~V}$ in the quantum wells using the commercial tool [9]. The lower hole density in the wells at room temperature also reduces the voltage across the $\mathrm{Si}$ cap and increases the electron tunneling current from Pt to semiconductor. However, for Al-gate devices at negative gate bias, due to the much smaller electron barrier $(3.1 \mathrm{eV})$ compared to the hole barrier $(4.7 \mathrm{eV})$, the electron current is dominant, and the electrons tunneling from the $\mathrm{Al}$ electrode are limited by the diffusion process (temperature dependent) in the p-type semiconductor. At $300 \mathrm{~K}$, the current of the SiGe dot device is similar to that of the Si device (Fig. 4 inset) due to electron current not being lowered by the repulsive barrier in the valence band. However, at $40 \mathrm{~K}$, the current decrease of both the SiGe dot device and the Si device compared to that at $300 \mathrm{~K}$ is not due to the repulsive barrier, but due to the temperature-dependent electron diffusion.

\section{CONCLUSION}

The transport mechanisms in SiGe dot MOS devices with $\mathrm{Al}$ and Pt gates are investigated. The valence band offset in $\mathrm{Si} / \mathrm{SiGe}$ heterojunction can confine holes and forms a repulsive energy barrier to block the hole current. In view of its application, the supply of holes from Pt into SiGe dots can be designed for the intraband transition in far infrared photodetectors [10].

\section{ACKNOWLEDGMENT}

The authors would like to thank Hung for the English improvement.

\section{REFERENCES}

[1] C. W. Liu, M. H. Lee, M.-J. Chen, I. C. Lin, and C.-F. Lin, "Roomtemperature electroluminescence from electron-hole plasmas in the metaoxide-silicon tunneling diodes," Appl. Phys. Lett., vol. 76, no. 12, pp. 1516-1518, 2000.

[2] C. W. Liu, W. T. Liu, M. H. Lee, W. S. Kuo, and B. C. Hsu, "A novel photodetector using MOS tunneling structures," IEEE Electron Device Lett., vol. 21, no. 6, pp. 307-309, Jun. 2000.

[3] M. H. Liao, C.-Y. Yu, T.-H. Guo, C.-H. Lin, and C. W. Liu, "Electroluminescence from the Ge quantum dot MOS tunneling diodes," IEEE Electron Device Lett., vol. 27, no. 4, pp. 252-254, Apr. 2006.

[4] B.-C. Hsu, S. T. Chang, T.-C. Chen, P.-S. Kuo, P. S. Chen, Z. Pei, and C. W. Liu, "A high efficient $820 \mathrm{~nm}$ MOS Ge quantum dot photodetector," IEEE Electron Device Lett., vol. 24, no. 5, pp. 318-320, May 2003.

[5] O. G. Schmidt and K. Eberl, "Multiple layers of self-assembled $\mathrm{Ge} / \mathrm{Si}$ islands: Photoluminescence, strain fields, material interdiffusion, and island formation," Phys. Rev. B, Condens. Matter, vol. 61, no. 20, pp. 13721-13729, 2000.

[6] G. Schmidt and K. Eberl, "Strain and band-edge alignment in single and multiple layers of self-assembled Ge/Si and GeSi/Si islands," Phys. Rev. B, Condens. Matter, vol. 62, no. 24, pp. 16715-16720, 2000.

[7] B.-C. Hsu, W.-C. Hua, C.-R. Shie, K.-F. Chen, and C. W. Liu, "Growth and electrical characteristics of liquid-phase deposited $\mathrm{SiO} 2$ on Ge," Electrochem. Solid-State Lett., vol. 6, no. 2, pp. F9-F11, 2003.

[8] C.-H. Lin, B.-C. Hsu, M. H. Lee, and C. W. Liu, "A comprehensive study of inversion current in MOS tunneling diodes," IEEE Trans. Electron Devices, vol. 48, no. 9, pp. 2125-2130, Sep. 2001.

[9] Synopsys Sentaurus Tcad Simulator.

[10] C.-H. Lin, C.-Y. Yu, P.-S. Kuo, C.-C. Chang, T.-H. Guo, and C. W. Liu, "Delta-doped MOS Ge/Si quantum dot/well infrared photodetector," Thin Solid Films, vol. 508, no. 1-2, pp. 389-392, 2006. 